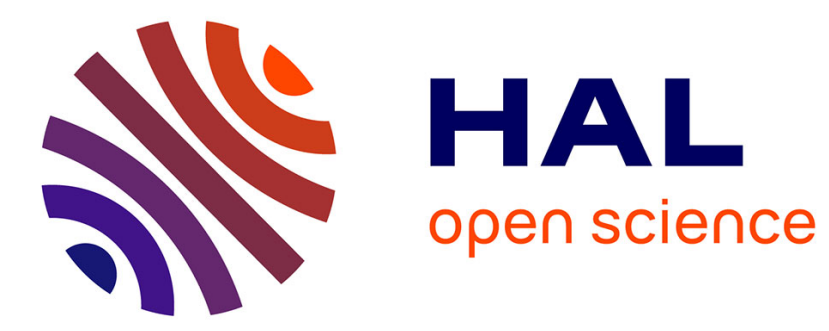

\title{
Les formes nominalisées du verbe dans quelques langues océaniennes
}

\author{
Claire Moyse-Faurie
}

\section{To cite this version:}

Claire Moyse-Faurie. Les formes nominalisées du verbe dans quelques langues océaniennes. Faits de langues, 2007, 30, pp.97-116. halshs-00559211

\section{HAL Id: halshs-00559211 https://shs.hal.science/halshs-00559211}

Submitted on 25 Jan 2011

HAL is a multi-disciplinary open access archive for the deposit and dissemination of scientific research documents, whether they are published or not. The documents may come from teaching and research institutions in France or abroad, or from public or private research centers.
L'archive ouverte pluridisciplinaire HAL, est destinée au dépôt et à la diffusion de documents scientifiques de niveau recherche, publiés ou non, émanant des établissements d'enseignement et de recherche français ou étrangers, des laboratoires publics ou privés. 


\title{
Les formes nominalisées du verbe dans quelques langues océaniennes
}

\author{
Claire Moyse-Faurie, Lacito CNRS
}

\section{PRÉSENTATION DES LANGUES PRISES EN COMPTE ET DE LA PROBLÉMATIQUE}

Il s'agira dans cet article $^{1}$ de comparer ce que peuvent être les formes nominalisées du verbe dans des langues océaniennes, en prenant principalement en considération les points suivants :

- dérivation nominale : procédés, valeurs et fonctions

- nominalisation de verbe versus nominalisation de syntagme ou de proposition

- combinatoire des formes nominalisées

- expression des actants

- fonctions syntaxiques

Les langues prises en compte dans cet article sont des langues océaniennes appartenant à différents sous-groupes: langues kanak de la Grande Terre (cèmuhî, xârâcùù, xârâgurè) et des îles Loyauté (drehu, nengone); fidjien ; langues polynésiennes (futunien, wallisien, tahitien, tokelau). Ce large éventail a pour but de mettre en évidence la corrélation entre le degré de différenciation catégorielle dans une langue donnée et le type de formes nominalisées qu'elle met en oeuvre. Pour cette raison, je commencerai par un bref rappel des problèmes que pose la catégorisation lexicale dans ces langues avant d'aborder les différents types de nominalisation.

\section{LA CATÉGORISATION SYNTAXIQUE}

L'opposition verbo-nominale est souvent délicate à définir dans les langues océaniennes car (i) ce sont très largement des langues omniprédicatives et (ii) il existe peu de variation morphologique des lexèmes en fonction du nombre, et aucune en fonction de la personne, du temps ou de l'aspect. Il n'existe par conséquent dans ces langues aucune forme du verbe correspondant à ce qu'on appelle infinitif, forme participiale ou gérondif. L'opposition verbo-nominale se

${ }^{1}$ Je tiens à remercier le comité de rédaction de Faits de Langues pour sa lecture attentive et ses judicieux commentaires. 
manifeste essentiellement dans les fonctions non prédicatives, mais à des degrés divers selon les langues. Dans les langues kanak de la Grande Terre, si tout lexème peut être prédicat, un verbe ne peut avoir d'autre fonction que la fonction prédicative à moins d'être dérivé. Ainsi, арии-döö "maître de la terre" est prédicat en (1), tout comme pètù "tresser" en (2) :
(1) Kwémwaa nää apuu-döö, kwé mê nê-kwââ Kwémwaa DuR.PsÉ maître-terre eau et feuille-arbre "Kwémwaa était maître de la terre, de l'eau et des feuilles."

(2) pâê nä pètù rè döxöu femme INACC tisser INACC natte "Les femmes tissent des nattes."

Bien entendu, арии-döö peut aussi avoir un emploi non prédicatif, comme par exemple sujet postposé en (3) :

$\begin{array}{lllll}\text { (3) è } & \text { wâ } & \text { toa } & \text { ngê } & \text { apuu-döö } \\ & \text { 3SG } & \text { ACCOM } & \text { arriver } & \text { M.SuJ chef-terre }\end{array}$ 3SG ACCOM arriver M.SuJ chef-terre
"Le maître de la terre est arrivé."

Par contre, pètù doit être dérivé pour référer à une entité et avoir une fonction autre que prédicative :

(4) döunämè nâ sii söpuru na kööxâ-râ, nâ xwigwéré môrô

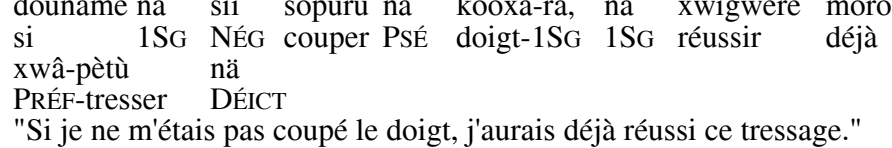

Dans les langues polynésiennes occidentales, la polyfonctionnalité est généralisée et tout lexème peut, sans subir un processus dérivationnel, assumer une fonction prédicative ou non prédicative. Cependant, les lexèmes référant à des procès employés non prédicativement ne sont pas compatibles avec l'ensemble des déterminants liés au contexte non prédicatif (cf. Moyse-Faurie 2005). Par exemple, dans l'énoncé futunien suivant, seul l'article spécifique le peut déterminer le syntagme non prédicatif dont le centre (ano "aller") réfère prototypiquement à un procès :

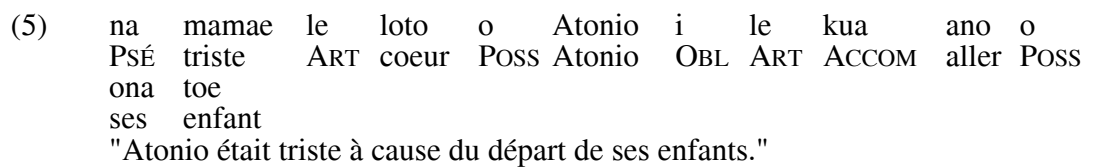

Les autres articles (pluriel ou non spécifiques) sont exclus dans cette position. 
Ainsi, on assiste au paradoxe suivant : d'une part, les lexèmes sont, à quelques exceptions près, invariables selon le nombre ${ }^{2}$, le temps, le mode, l'aspect ou la personne, et il n'existe pas de genre lexicalisé ; d'autre part, l'opposition verbonominale est essentiellement basée sur des orientations sémantiques différentes selon l'emploi syntaxique et non sur des incompatibilités fonctionnelles. Or, les procédés dérivationnels restent importants, même quand ils ne sont pas nécessaires à l'emploi non prédicatif de lexèmes référant à des procès.

J'examinerai en détail à quoi servent ces procédés dérivationnels, quel est l'impact sur la catégorisation et les possibilités combinatoires. Auparavant, il est nécessaire d'établir une distinction importante entre création lexicale à partir de verbes dérivés et phrases nominalisées, étant donné que, dans toutes les langues prises en compte ici, les mêmes procédés de nominalisation peuvent avoir ces deux fonctions.

\section{LES DIFFÉRENTS TYPES DE "FORMES NOMINALISÉES"}

Que peut-on entendre par "formes nominalisées du verbe" dans des langues océaniennes ? S'agit-il de procédés dérivationnels transformant un verbe en nom, nouvelle unité lexicale, ou de l'emploi d'un lexème habituellement prédicat dans un syntagme non prédicatif ? Dans les langues de Nouvelle-Calédonie, les stratégies se recoupent. Ainsi, en xârâcùù, le préfixe ù- "lieu de, cause de" peut nominaliser des verbes pour former une nouvelle entité :

$$
\begin{array}{ll}
\text { ù-sêê } & \text { ù-fädë } \\
\text { PRÉF-bifurquer } & \text { PRÉF-marcher } \\
\text { "bifurcation" } & \text { "traces de pas" }
\end{array}
$$

Un verbe nominalisé par préfixation peut s'adjoindre un déterminant possessif, le syntagme obtenu pouvant constituer un circonstant autonome, à valeur causale en (7) :

$$
\begin{array}{lllll}
\text { (7) nâ } & \text { kwèti } & \text { ù-sööpö } & \text { rè } & \text { xöu } \\
\text { 1SG } & \text { être fatigué } & \text { PRÉF-laver } & \text { Poss } & \text { linge } \\
& \text { "Je suis fatiguée d'avoir lavé le linge" } &
\end{array}
$$

Dans les langues océaniennes, les mêmes procédés de dérivation peuvent donner lieu à plusieurs degrés de nominalisation :

a) La nominalisation affecte un seul lexème verbal, et donne naissance à un nouveau lexème appartenant à la catégorie nominale. Le dérivé constitue une seule entité lexicale, présente toutes les latitudes combinatoires d'un nom, et

2 Dans les langues polynésiennes occidentales en particulier, la première syllabe de quelques prédicats intransitifs est rédupliquée lorsque l'actant à l'absolutif réfère à une pluralité. 
seulement du nom ; les fonctions du lexème nominalisé sont celles du nom. Ce type de nominalisation a une productivité variable, et donne naissance à des formes idiosyncratiques par rapport au verbe dont elles sont issues : leur sens n'est pas prévisible.

b) La nominalisation affecte un syntagme verbal ou une proposition, mais donne, ici aussi, naissance à une seule entité lexicale, quoique complexe, comme dans le composé drehu (8b), obtenu à partir de la phrase verbale (8a) constituée du prédicat kei, d'un actant introduit par la marque sujet hnei et d'un circonstant :

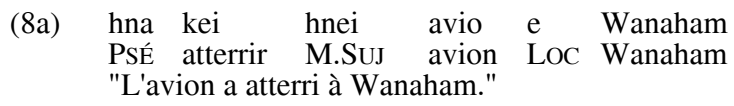

L'article déictique la nominalise l'ensemble, composé d'une unité lexicale complexe, hna kei hnei avio "aéroport" et de son expansion locative $e$ Wanaham:

(8b) la hna kei hnei avio e Wanaham "l'aéroport de Wanaham" (L. Sam, com. pers.)

Cette unité lexicale peut être pluralisée (itre, marque pluriel) : (8c) la itre hna kei hnei avio
"les aéroports" (id.)

La structure interne du dérivé reste identique à celle d'un syntagme prédicatif. Cependant, le dérivé ne réfère plus qu'à une seule entité, sorte de composé périphrastique.

Toujours en drehu, le composé sine ce tro (compagnon/ensemble/aller) "compagnon de route" peut être pluralisé : la itre sine ce tro "les compagnons de route", mais la nominalisation est seulement partielle car la possession ne peut être exprimée que par un complément accompagnatif, et non par un déterminant possessif :
(9a) meköle hë la lue sine ce tro me eni dormir ACCOMART deux compagnon ensemble aller COM $1 \mathrm{SG}$ "Mes deux compagnons de route se sont endormis."

Seule la forme pleinement nominalisée par l'adjonction du suffixe -nge accepte un déterminant possessif introduit par la marque $i$ :

$\begin{array}{llllll}\text { (9b) Wamo la } & \text { sine } & \text { tro-nge } & \text { i } & \text { eni } \\ \text { Wamo ART compagnon aller-SUFF } & \text { Poss } & 1 \text { SG } \\ \text { "Wamo est mon compagnon de route." } & & \end{array}$ 
De même, l'énoncé drehu suivant comporte un syntagme nominalisé dont le centre nyi $i$ (litt. "faire poisson") signifie "pêche, pêcher" et fonctionne comme une unité lexicale, avec un déterminant possessif $i$ angatr. En outre, le syntagme nominalisé peut être pluralisé :

(10a) the qaja kö la itre nyi $\mathrm{i} \quad$ i $\quad$ angatr PROH dire INT ART PL faire poisson POSS 3PL "Ne parle pas de leurs pêches."

c) La nominalisation affecte une proposition qui devient un syntagme actanciel ou circonstanciel. Sémantiquement, la forme nominalisée réfère toujours à l'événement - la nominalisation est sémantiquement prévisible - et ne constitue pas une nouvelle entité lexicale. Les expansions sont, comme dans le cas précédent, de différents types, soit actanciel soit possessif, mais la combinatoire et les fonctions possibles peuvent être plus restreintes que celle des noms.

Il est ainsi possible de nominaliser la proposition tro angatr a nyi $i$ "ils vont aller pêcher" en y antéposant l'article déictique $l a$ :

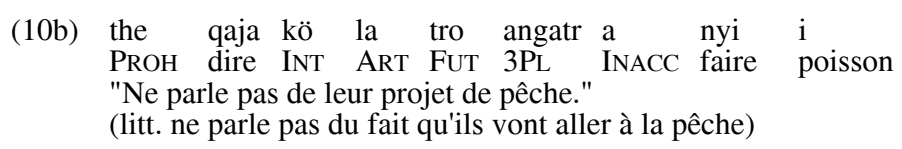

Mais il est impossible de pluraliser cette proposition nominalisée à l'aide du morphème du pluriel itre : *the qaja kö la itre tro angatr a nyi i (qui signifierait "ne parle pas de leurs projets de pêche"), alors que la pluralisation d'un syntagme nominalisé est possible, comme exemplifiée en (10a).

Par conséquent, les critères pour distinguer une forme nominalisée d'une forme nominale diffèrent selon que la dérivation est un simple procédé de création lexicale ou un procédé grammatical. Dans le premier cas, rien ne différencie un dérivé nominal et un nom, tous deux n'ayant qu'un seul référent ; dans le second, les possibilités combinatoires sont plus restreintes et les référents à l'intérieur de la proposition nominalisée restent distincts.

Dans cet article, je prendrai essentiellement en compte le procédé grammatical, à savoir la nominalisation de propositions qui n'aboutit pas à la formation d'une unité lexicale complexe.

\section{LES DEUX GRANDS TYPES DE PROPOSITIONS NOMINALISÉES : LANGUES À} ARTICLE VERSUS LANGUES À AFFIXE

Pour nominaliser une proposition, les langues océaniennes prises en compte ici utilisent deux grands procédés (qui ne sont pas nécessairement exclusifs l'un de l'autre) et peuvent par conséquent être classées en deux types : 
a) les langues à article (langues des îles Loyauté, fidjien, langues polynésiennes), dans lesquelles l'article nominalise la phrase verbale par sa seule présence $^{3}$. Dans ces langues, la dérivation par affixation est avant tout productrice de lexèmes et n'est pas nécessaire à la nominalisation de proposition. Dans ce type de langues, le choix de l'article entraînant la nominalisation du syntagme ou de la proposition est contraint.

b) Dans les langues kanak de la Grande Terre, c'est avant tout la présence d'un préfixe sur le verbe nominalisé qui marque la nominalisation de la proposition, comme en xârâcùù :

$\begin{array}{lllllll}\text { (11) tö } & \text { a } & \text { è } & \text { dù } & \text { kèè-toanôô } & \text { röö } & \text { niè } \\ \text { LoC } & \text { ici } & 3 \mathrm{SG} & \text { possible } & \text { PRÉF-trouver } & \text { PosS+2SG } & 3 \mathrm{SG}\end{array}$ LOC ici 3SG possible PRÉF-trouver POSS+2SG 3SG
"C'est ici que tu peux le trouver" (litt. ici il est possible ton trouvage lui)

Cependant, le syntagme nominalisé peut parfois comporter à la fois un préfixe et un article, comme dans l'exemple (12) en cèmuhî (Rivierre 1980:277), le préfixe $a$ - indiquant le lieu où se déroule l'action (correspondant à la phrase verbale : lé taa-bé /3PL/monter-DIR/ "ils montent en s'approchant") :

$\begin{array}{llll}\text { (12) } & \text { a } & \text { a-taa-bé } & \text { tè-lé } \\ & \text { ART } & \text { PRÉF-monter-DIR } & \text { POSS-3PL }\end{array}$

"leur trajet pour monter" (litt. le lieu de monter à eux en s'approchant)

De même en drehu avec à la fois l'article déictique la et le préfixe hna- :

(13) tuun la hna-up hnei maaseta i thu comme ART PRÉF-envoyer M.Suj maître POSS lézard
"...pareil à l'envoi du maître du lézard."

\section{LES COMPOSANTES DE LA PROPOSITION NOMINALISÉE}

\subsection{L'article}

Dans les langues polynésiennes et en fidjien, l'article suffit à lui seul à nominaliser un lexème, un syntagme ou une proposition. Mais si tout lexème est compatible avec l'ensemble des articles, déictiques ou autres déterminants du syntagme non prédicatif, le choix de l'article est restreint lorsqu'il s'agit de nominaliser un syntagme ou une proposition dont le centre réfère à un procès. Dans ce cas, seul l'article singulier spécifique ou défini (tongien $e / h e$; futunien, samoan le; wallisien, tokelau, tahitien $t e$, etc. ; fidjien $n a \sim a$ ) peut introduire la structure nominalisée, comme dans l'exemple wallisien suivant :

3 Ce procédé n'est pas propre aux langues océaniennes. Koptjevskaja-Tamm (1993:50) donne le lakhota comme exemple de ce type de langues. 
(14) Kua vaha'a lua nei te kua fakahifo o te ACCOM semaine deux maintenant ART ACCOMdescendre POSS ART 'aliki hau o Alo e tana fâmili. chef roi Poss Alo ERG sa famille

"Il y a maintenant deux semaines qu'a eu lieu la destitution du roi de Alo par sa famille."

De même en fidjien, Dixon (1988:130-131) note la présence obligatoire de l'article commun $a / n a$ en tête de la proposition nominalisée :

(15) au maarau va'alevu i na omudou yaco mai qoo $1 \mathrm{SG}$ heureux très à ART votre arriver DIR DEIC "Je suis très heureux de votre arrivée ici."

En nengone (îles Loyauté), l'article singulier défini et spécifique ore introduit le syntagme nominalisé, ici aussi sans affixe dérivationnel sur le verbe :

(16) ore hue ni Waetea hna a-cengenia-ni ore node ART partir POSS Waetea PSÉ CAUS-colère-TRANS ART gens "Le départ de Waetea a mis en colère les gens."

(17) ore ru(e) ael bon, sesako

ART faire bounia $3 \mathrm{SG}_{\mathrm{G}}$ drôle

"Le fait qu'il prépare le bounia est drôle." (litt. son faire bounia est drôle)

En futunien, c'est aussi l'article spécifique le qui introduit les phrases nominalisées :

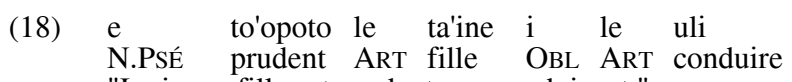
"La jeune fille est prudente en conduisant."

Et en tahitien, l'article spécifique te a la même fonction :

(19) $\mathrm{Na}$ hea te tatara i te reira mau fifi ?
par où ART résoudre OBL ART ANAPH PL problème
"Comment résoudre ces problèmes?" (Vernaudon et Rigo 2004:467)

Les adjectifs possessifs, incluant dans leur formation un article amalgamé, peuvent aussi nominaliser une proposition si l'article est spécifique singulier, comme dans l'exemple futunien (20) avec le possessif lana "sa, son", composé de l'article $l-(<l e)$, du relateur possessif $a$ (possession aliénable) et de la 3ème personne du singulier $-n a$ :

(20) na tu'ā a Soane e le kau aliki i lana ano o māta'u PsÉpunir ABS Soane ERG ART COLL chef OBL son aller pour pêcher "Les chefs ont puni Soane d'être allé pêcher à la ligne."

(litt. ... de son [fait d']aller pour pêcher) 


\subsection{L'affixe}

a) Les préfixes nominalisants des langues kanak

Dans les langues kanak de la Grande Terre, plusieurs préfixes, chacun au sémantisme bien particulier, peuvent tout à la fois introduire des syntagmes nominalisés et créer des dérivés nominaux. En xârâcùù, la nominalisation peut s'effectuer à l'aide de l'un des préfixes suivants : kèè- "façon, manière" (le plus neutre) ; ̀̀- "lieu, cause" ; $x w \hat{a}-$ "fait, occasion", $a$ - (SG)/pa- (pl) "nom d'agent", êe- "résultat", etc.

Des dérivés peuvent être formés à partir de verbes statifs et réfèrent alors à la qualité, comme en xârâgurè le verbe xöru "[être]bon" (21a), dérivé en kèè-xöru "la bonté" (21b) :

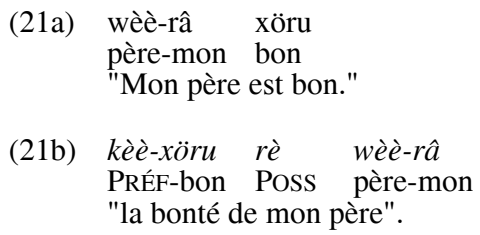

Les verbes actifs admettent généralement plusieurs préfixes. Ainsi, le verbe pùùtè "se rencontrer", prédicat dans l'exemple suivant :
(22a) dèèri mê anyââ pùùtè tö Kwênyêngêrê gens et maman se rencontrer LoC Kwênyêngêrê "Les gens et maman se rencontrent à Kwênyêngêrê.

peut donner lieu à différents dérivés : kèè-pùùtè "manière de se rencontrer", pùùtè "lieu de rencontre", puu-pùùtè "raison ayant provoqué la rencontre", kêrêpùùtè "thème de la rencontre", êrê-pùùtè "ordre du jour de la rencontre", xêêpùùtè "possibilité de rencontre" ou encore xwâ-pùùtè "rencontre, occasion de se rencontrer", comme dans l'exemple (22b) :

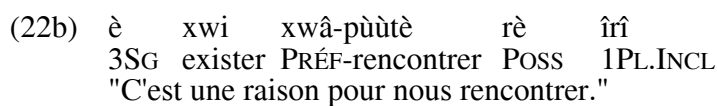

b) Les suffixes nominalisants des langues polynésiennes

Les suffixes nominalisants des langues polynésiennes sont moins variés sémantiquement que ne le sont les préfixes des langues kanak; ils indiquent essentiellement le lieu ou le résultat d'une action. D'autre part, la dérivation n'est pas nécessaire à l'emploi de propositions nominalisées, bien qu'elle puisse y être associée, dans des conditions souvent difficiles à déterminer. 
Selon Chung (1973), il faut distinguer en maori deux types de nominalisation : avec l'un, qui ne nécessite pas de suffixe, on obtient des formes nominalisées qui décrivent un résultat ou une qualité abstraite ; avec l'autre, nécessitant un suffixe (reflet du Proto-polynésien $*(\mathrm{C})(\mathrm{a})$ a), la forme nominalisée exprime l'occurrence d'une action ou d'un état.

Clark (1981) conteste, à juste titre nous semble-t-il, cette analyse, en montrant que des constructions sans suffixe en maori expriment tout à fait l'occurrence d'une action ou d'un état. Selon Clark, la présence du suffixe serait déterminée par le contexte syntaxique large dans lequel la nominalisation s'effectue.

Hooper (1996) propose une analyse très fine de la présence ou absence de suffixe dans les formes nominalisées du tokelau. Les formes suffixées seraient utilisées pour des situations actualisées (aspect realis), avec des verbes téliques comportant deux actants dont l'actant objet spécifique, comme en (23a) :

Tokelau (Hooper 1996:230)

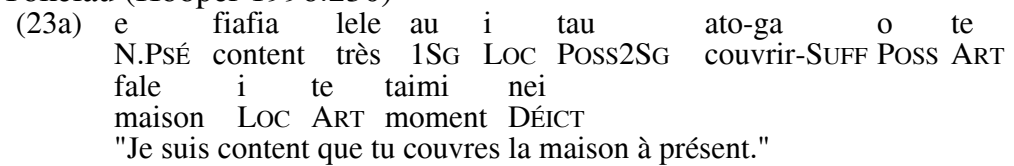

D'après Hooper (1996), les formes nominalisées sans suffixe référeraient à des concepts abstraits, à l'aspect irrealis, essentiellement pour des actions atéliques ou habituelles, avec un actant objet qui soit non spécifique ou incorporé comme en $(23 b)$ :

(23b) he gāluega faigatā te vali fale
ART travail difficile ART peindre maison
"Peindre une maison est un travail difficile."

Les langues qui ne font pas de distinction entre ces deux types de nominalisation conservent cependant l'usage du suffixe pour former des dérivés lexicaux décrivant le lieu d'une action ou des termes abstraits.

\subsection{Les marques aspectuelles et temporelles}

La compatibilité des aspects dans les syntagmes nominalisés est attestée aussi bien dans les langues polynésiennes qu'en fidjien et dans les langues kanak, comme par exemple en cèmuhî :

$$
\begin{array}{lllll}
\text { Cèmuhî (Rivierre 1980:268) } & & & \\
\text { (24) pa } & \text { a-caa-tupwö } & \text { olè hê } & \text { ali } & \text { pwön } \\
\text { ART.MASC PRÉF-ACCOM-tomber } & \text { en bas dans } & \text { ART.NeUtRE } & \text { trou } \\
\text { "l'homme tombé dans le trou" } & & & &
\end{array}
$$


En outre, dans les langues kanak et en fidjien, la nominalisation n'exclut aucune des marques temporelles, comme la marque du passé dans les exemples suivants :

Xârâcùù (Moyse-Faurie 1995)

(25) ù-fa-céé
PRÉF-CAUS-accoster PSÉ Pò Po pêcheur bateau
"lieu où le pêcheur avait fait accoster le bateau"

Fidjien (Dixon 1988:132)

(26) au tadra-a a omu aa la'o mai 1SG rêver-TRAns ART ton PSÉ aller DIR "J'ai rêvé que tu étais venu."

En futunien (Moyse-Faurie 2005), le verbe nominalisé par l'antéposition de l'article le en (27) garde sa compatibilité avec l'aspect continu koi, ainsi que son redoublement partiel (nofo $>$ nonofo) marquant la pluralité des participants :

(27)
e
'ita
a Soane i
le koi
nonofo
N.PSÉ (être en) colère ABS Soane OBL ART CONT RÉDUP.rester
a Malia mo lona tinana.
Poss Malia et sa mère
"Soane est en colère parce que Malia est encore avec sa mère."
(litt. Soane est en colère à cause de l'encore rester de Malia et sa mère)

Cependant, dans les langues polynésiennes, seuls les aspects peuvent faire partie du syntagme nominalisé, les marques temporelles du passé et du nonpassé en sont exclues.

\subsection{Autres compatibilités}

Les adverbes, qu'ils soient grammaticaux ou lexicaux, de même que les auxiliaires verbaux peuvent conserver leur place à côté du verbe nominalisé. Ainsi, en xârâgurè, xaari "seul" est constitutif de l'énoncé prédicatif :

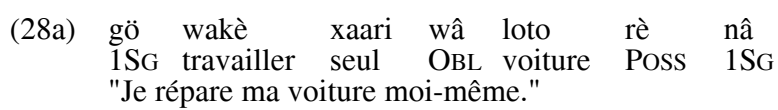

comme du syntagme nominalisé :
(28b) gö tômwâ kèè-wakè xaari rè wâ loto rè nâ 1SG savoir PRÉF-travailler seul Poss OBL voiture Poss $1 \mathrm{Sg}$ "Je sais réparer ma voiture moi-même."

En wallisien, l'auxiliaire verbal fia "avoir envie" conserve sa position, immédiatement avant le verbe, dans le syntagme nominalisé : 
(29) kua haga ia o kai te mo'i pane $i$ tona kua ACCOM faire face 3SG COMP manger ART CLAS pain OBL son ACCOM fia kai

avoir envie manger

"Il s'était mis à manger le morceau de pain tant il avait faim."

(litt. ... à cause de son avoir eu envie de manger)

De la même façon, le postverbe directionnel centripète mai "en venant de" se maintient dans le syntagme à centre verbo-nominalisé :

(30) 'E au nofo pē anai i hen̄̄ o a'u ki te hoki N.PsÉ 1SG rester RESTR FUT Loc ici jusqu'à ART IMM liliu mai a koutou retourner DIR POSS 2PL

"Je resterai ici jusqu'à votre imminent retour."

\section{L'EXPRESSION DES ACTANTS}

\subsection{Dans les langues kanak}

L'expression des actants dans la structure nominalisée dépend de leur rôle sémantique ainsi que de la catégorie et de la valence du verbe dérivé. Dans certains cas, l'ancien actant conserve sa marque actancielle mais le plus souvent il devient un déterminant possessif.

En xârâcùù, le déterminant d'un verbe intransitif nominalisé (correspondant au sujet de l'énoncé verbal) est introduit par la marque possessive rè, que ce soit un pronom ou un nom :

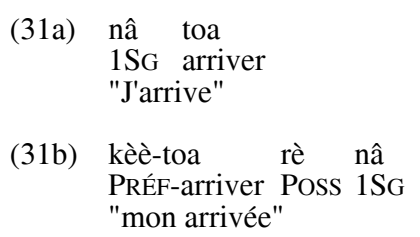

kwâ toa
bateau arriver
"Le bateau arrive."

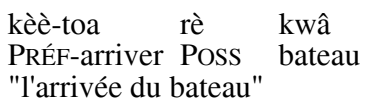

Si le verbe est transitif, comme en (32a) :

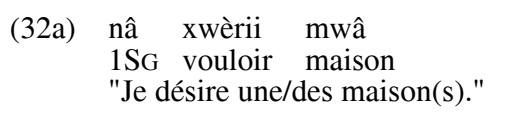

il peut être nominalisé et s'adjoindre : 
- soit un déterminant correspondant au sujet de l'énoncé verbal ; sémantiquement, c'est un expérient, marqué syntaxiquement comme un possesseur :

$\begin{array}{lll}\text { (32b) } & \text { kèè-xwèrii } \\ & \text { PRéF-vouloir nâ } \\ \text { "mon désir" } & \text { Poss } 1 \text { SG }\end{array}$

- soit un déterminant correspondant à l'objet de l'énoncé prédicatif, et introduit également par le relateur possessif rè :

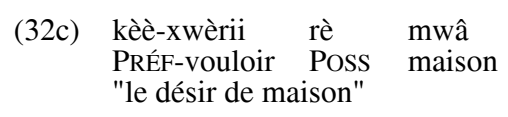

- soit les deux déterminants exprimés conjointement : celui qui correspond sémantiquement au patient est alors juxtaposé au syntagme possessif référentiellement expérient :

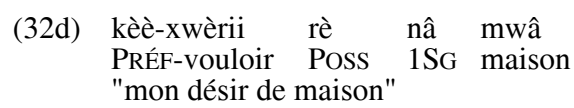

Autre exemple, toujours en xârâcùù, formant un énoncé complet, avec deux actants exprimés, l'un sous forme de déterminant possessif (l'agent), l'autre simplement juxtaposé (le patient) :

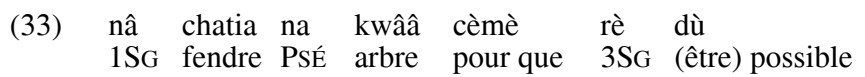

kèè-chuè rè nâ nè

PRÉF-souffler Poss 1SG feu

"J'ai fendu du bois pour pouvoir allumer mon feu"

(litt. pour que soit possible mon allumage feu)

Le déterminant juxtaposé référant au patient peut être un pronom : c'est alors la forme "indépendante" du pronom qui apparaît, et jamais la forme possessive :

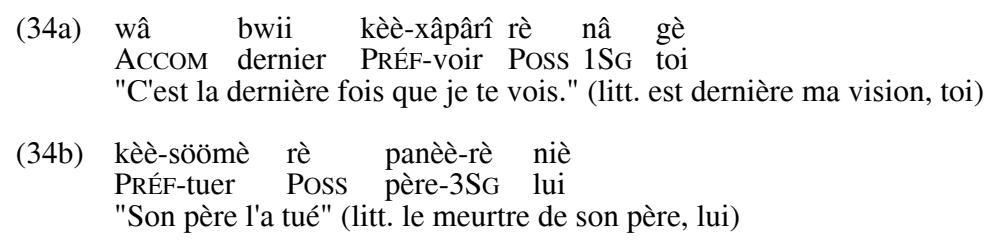

A l'inverse, si le père est le patient et le fils l'agent, on obtient l'énoncé suivant : 
(34c) kèè-söömè rèè panèè-rè

PRÉF-tuer Poss+3Sg père-3SG

"Il a tué son père" (litt. son meurtre, son père)

Cette impossibilité pour le verbe nominalisé d'avoir deux déterminants possessifs à sa droite est constante dans toutes les langues de notre corpus. Lorsque les deux participants sont exprimés, seul l'agent est morphosyntaxiquement marqué comme déterminant, introduit par la marque possessive rè ; lorsque l'agent n'est pas exprimé, le patient prend sa position dans la chaîne parlée, comme en (32c).

Selon la typologie de de Koptjevskaja-Tamm (1993:61) la construction nominalisée du xârâcùù est en partie de type "Possessive-Accusative", avec l'unique actant (S) et l'actant référant à l'agent du verbe transitif (A) tous deux au génitif ; cependant, l'actant référant au patient $(\mathrm{O})$ ne garde pas le statut d'objet qu'il a dans l'énoncé verbal, puisque c'est la forme indépendante du pronom qui apparaît dans la structure nominalisée.

En xârâcùù, dans un énoncé prédicatif, la thématisation du sujet par postposition se fait à l'aide du relateur ngê. Il est aussi possible de thématiser l'agent des syntagmes à verbe transitif nominalisé, à l'aide du même relateur :

\footnotetext{
(35) tö a è dù kè̀̀-toanôô rèè ngê gè LOC ici 3Sg possible PRÉF-trouver POSS+3Sg M.SUJ 2SGINDÉP "C'est ici que toi, tu peux le trouver"

(litt. ici il est possible son trouvage, toi impliqué)
}

Le patient est alors le déterminant du syntagme possessif, la périphérisation de l'agent ayant pour effet de lui laisser sa place.

C'est ce type de construction que l'on retrouve en nêlêmwa, avec l'actant pronominal objet (référant au patient) suffixé au verbe nominalisé, tandis que l'actant référant à l'agent est périphérisé (Cf. Bril 2002:76, exemples 10 et 11). Toujours selon la typologie de Koptjevskaja-Tamm (1993:61), cette construction relève du sous-type "Oblique-Possessive" du type "Ergative-Possessive", avec l'unique actant $(S)$ et le patient d'un verbe transitif $(P)$ au génitif, tandis que le patient d'un verbe transitif est à l'oblique.

\subsection{Dans les langues polynésiennes : l'exemple du wallisien}

Dans les langues polynésiennes également, un verbe nominalisé ne peut jamais avoir plus d'un déterminant possessif postposé (Clark 1981, MoyseFaurie 2000). Dans toutes ces langues (à l'exception du niue), il existe deux types de possession: l'une, qualifiée d'agentive ou d'aliénable, utilise la préposition $a$; l'autre, objectale, inaliénable, se construit à l'aide de la préposition $o$. Les adjectifs possessifs, constitués d'un article, d'une préposition possessive et d'une marque de personne, présentent ainsi deux formes selon le 
type de possession ; par exemple, en wallisien, taku (forme agentive) et toku (forme patientive) pour l'adjectif possessif singulier spécifique de $1^{\text {ère }}$ personne.

Examinons en détail la situation en wallisien, langue ergative dans laquelle il existe trois catégories principales de verbes : intransitifs, transitifs indirects et transitifs ergatifs.

\subsubsection{Nominalisation de verbes intransitifs}

Les verbes intransitifs ont leur actant unique à l'absolutif (marque $\varnothing$ devant un nom précédé d'article, ia devant un nom propre ou un pronom) et sont, sur le plan sémantique, soit orientés patient, soit orientés agent. En devenant centre de groupe non prédicatif, l'actant à l'absolutif d'un verbe intransitif orienté patient devient déterminant possessif en $o$ tandis que celui d'un verbe intransitif orienté agent devient déterminant possessif en $a$ :

Verbe orienté patient

$$
\begin{aligned}
& \text { (36a) 'e tā te pele } \\
& \text { N.PsÉ frapper ART cloche } \\
& \text { "La cloche sonne." }
\end{aligned}
$$

Verbe orienté agent

$$
\begin{aligned}
& \text { (37a) 'e lele te hosi } \\
& \text { N.PSÉ courir ART cheval } \\
& \text { "Le cheval court." }
\end{aligned}
$$

\section{Possession en $o$ de l'actant}

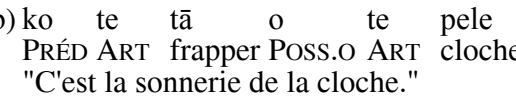

Possession en $a$ de l'actant

$$
\begin{aligned}
& \text { (37b) ko te lele a te hosi } \\
& \text { Pred ART course Poss.a ART cheval } \\
& \text { "C'est la course du cheval." }
\end{aligned}
$$

Il faut parfois prendre aussi en considération la place qu'occupe l'actant sur le gradient animé inanimé, ce dernier pouvant être, dans la structure nominalisée, introduit soit par la préposition $o$ soit par la préposition $a$, comme c'est le cas avec tagi "pleur, pleurer". En (38a), le déterminant possessif fāgana "légende" est perçu comme un patient, tandis qu'en (38b), telefoni "téléphone" est marqué comme un agent avec la marque possessive aliénable/agentive $a$ :
(38a) ne'e mole ina logo'i te tagi o te fāgana
PSÉ NÉg 3Sg entendre ART pleurer POSS.O ART légende
"Il n'a pas entendu le refrain de la légende." (litt. le pleur de la légende)
(38b) ne'e mole ina logo'i te tagi $a$ te telefoni
PSÉ NÉG 3SG entendre ART pleurer POSS.A ART téléphone
"Il n'a pas entendu sonner le téléphone." (litt. le pleur du téléphone)

\subsubsection{Verbes transitifs indirects}

Un verbe transitif indirect a, outre un actant à l'absolutif, un actant oblique introduit par la préposition ki (kiā devant pronom ou nom propre). Quand un 
verbe transitif indirect est nominalisé, son actant à l'absolutif est le plus souvent marqué par $a$.

(39) ko taku hoki sio ki te me'a fênei
PRÉD POSS.A.1SGIMM voir OBL ART chose être ainsi
"C'est la première fois que je vois une chose pareille."

Cependant, le choix entre les deux types de possession reste possible avec les verbes de sentiment, comme 'ofa "aimer", 'ita "être en colère", fehi'a "détester", etc., marquant alors la différence entre un sentiment éprouvé involontairement, passivement (40a avec la forme patientive tou du possessif), et un sentiment dont on est conscient et qu'on entretient (40b) avec la forme agentive tau du possessif) :

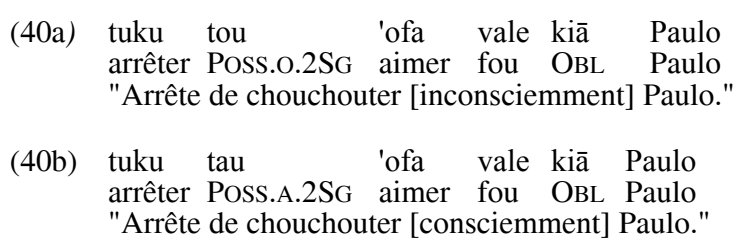

Notons que l'actant oblique ne peut jamais devenir le déterminant possessif du verbo-nominal.

\subsubsection{Verbes transitifs ou ergatifs}

Le verbe transitif/ergatif peut avoir, outre un actant à l'absolutif (référentiellement le patient), un actant à l'ergatif, introduit par la préposition $e$ et référant à un agent. Etant donné que le verbo-nominal n'a qu'une seule place de déterminant possessif à offrir, plusieurs cas de figure sont à considérer selon la catégorie grammaticale des actants.

a) Les deux actants sont des pronoms

Il y a choix entre un déterminant possessif en $a$ correspondant à l'actant ergatif "agent" comme en (41a) avec le possessif agentif tana, ou entre un déterminant possessif en $o$ correspondant à l'actant absolutif "patient" comme en (41b) avec le possessif tou. L'actant qui ne devient pas déterminant possessif garde ses marques actancielles absolutive (41a) ou ergative (41b) :
(41a) ne'e mole ke 'alu ko tana le'ole'o ia koe
PsÉ NÉG 2SG aller Préd Poss.A.3Sg surveiller ABs 2SG
"Tu n'es pas parti parce qu'il t'a surveillé."
(litt. ...sa [agent] surveillance toi)
(41b) ne'e mole ke 'alu ko tou le'ole'o e ia 
PSÉ NÉg 2Sg aller Préd Poss.o.2Sg surveiller ERg 3Sg

"Tu n'es pas parti parce qu'il t'a surveillé."

(litt. ...ta [patient] surveillance par lui)

b) L'un des actants est un pronom, l'autre est un nominal

Si l'actant à l'ergatif, référentiellement l'agent, est un nominal, il reste obligatoirement introduit par la marque ergative $e$, tandis que l'actant à l'absolutif prend la forme d'un possessif en $o$ :

(42) ko tona tāmate'i e Paulo..

PRÉD POSS.O.3Sg frapper ERG Paulo

"le fait que Paulo l'ait frappé" (litt. son [patient] frappage par Paulo)

(43) ko tou le'ole'o e te tagata faiako...

PRÉD POSS.O.2SG surveiller ERG ART homme enseigner

"la surveillance exercée sur toi par le professeur"

(litt. ta [patient] surveillance par le professeur)

Si c'est l'actant à l'absolutif qui est nominal, deux possibilités lui sont offertes :

- soit il devient déterminant possessif en $o$, placé immédiatement après le verbo-nominal, et l'actant pronominal référant à l'agent reste à l'ergatif :
(44) ko te mole kei tō o te kava e ia
Préd ARt NÉG Rém planter Poss.o ART kava ERG 3SG
"C'est un fait qu'il ne plante plus du kava."

- soit c'est l'actant pronominal référant à l'agent qui devient déterminant possessif en $a$ et l'actant nominal reste à l'absolutif. Ainsi, en (45b), le possessif tau réfère à l'agent du procès kai "manger" et correspond à l'actant pronominal antéposé en (45a), tandis que te mo'i mei est à l'absolutif dans les deux énoncés :

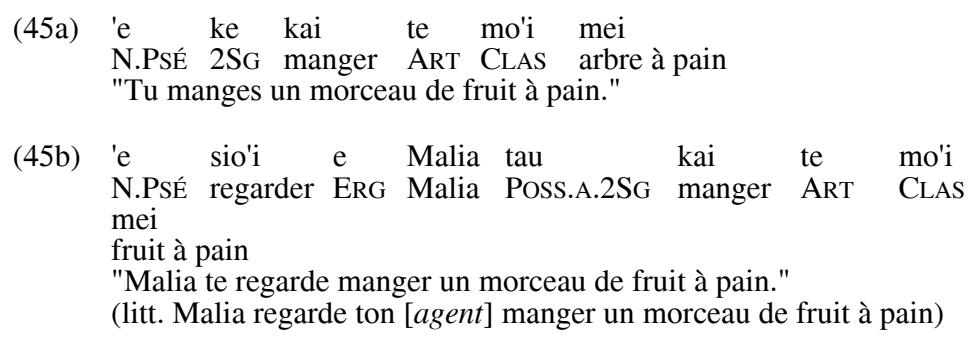

De même, l'actant pronominal référant à l'agent en (46a) est exprimé sous la forme du possessif agentif taku en (46b), alors que l'actant référant au patient reste à l'absolutif : 
$\begin{array}{llll}\text { (46a) ne'e au fakamatuku'i ia } & \text { Soane } \\ \text { PSÉ } 1 \text { SG effrayer } & \text { ABS Soane }\end{array}$

"J'ai fait peur à Soane."

(46b) ne'e hola te kulī ko taku fakamatuku'i ia Soane PSÉ fuir ART chien PRÉD POSS.A.1SG effrayer ABS Soane "Le chien s'est enfui quand j'ai fait peur à Soane."

(litt. le chien s'est enfui, c'est mon [agent] faire peur à Soane)

c) Les deux actants sont des nominaux

Dans ce cas, l'actant à l'ergatif conserve obligatoirement sa marque ergative, quelle que soit sa position, et l'actant à l'absolutif conserve ses deux positions possibles :

- il peut être déterminant possessif placé immédiatement après le verbonominal :

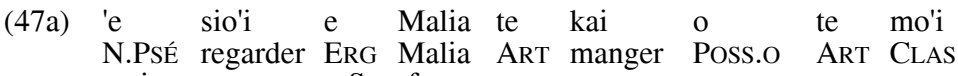
mei e Sosefo

fruit à pain ERG Sosefo

"Malia regarde Sosefo manger un morceau de fruit à pain."

(litt. Malia regarde le manger du morceau de fruit à pain par Sosefo)

- il peut rester à l'absolutif s'il est placé après l'actant à l'ergatif :
(47b) 'e sio'i e Malia te kai e Sosefo
N.PSÉ regarder ERG Malia ART manger ERG Sosefo
te mo'i mei
ART ClAS fruit à pain
"Malia regarde Sosefo manger un morceau de fruit à pain."

A titre de comparaison, en samoan, d'après Chung (1973:665), l'actant à l'absolutif serait, dans les deux positions, toujours exprimé sous la forme d'un déterminant possessif introduit par la préposition $o$, ce qui correspond à (47a).

d) Un seul actant est exprimé

Très souvent, un verbe ergatif n'est accompagné que d'un seul de ses deux actants potentiels. En wallisien, dans la structure nominalisée, le seul actant exprimé apparaît toujours comme un déterminant possessif, introduit par la préposition $a$ si c'est un agent (48a), et par la préposition $o$ si c'est un patient (48b). Ainsi, dans le contexte d'un verbe ergatif nominalisé, l'actant référentiellement agent, s'il est le seul actant exprimé, ne peut conserver sa marque ergative et est introduit par la marque possessive agentive $a$ (48a); de 
même, l'actant référentiellement patient, s'il est le seul actant exprimé, ne peut rester à l'absolutif et est introduit par la marque possessive patientive $o(48 \mathrm{~b})$ :
(48a) ko te le'ole'o a te tagata faiako
PRÉD ART surveiller POSS ART homme enseigner
"C'est la surveillance exercée par le professeur."
(48b) ko te le'ole'o o te tagata faiako
PRÉd ART surveiller POSS ART homme enseigner
"C'est la surveillance exercée sur le professeur"
(48c) *ko te le'ole'o e te tagata faiako, *ko te le'ole'o te tagata faiako

En contraste, la phrase verbale correspondante est, hors contexte, théoriquement ambiguë. En effet, le verbe $l e^{\prime}$ ole $^{\prime} o$ est un verbe labile; dans une construction intransitive, l'unique actant exprimé est à l'absolutif (marque $\varnothing$ en $(46 d))$ et peut référer aussi bien à un agent qu'à un patient :

$$
\begin{array}{llll}
\text { (48d) } & \text { 'e le'ole'o te tagata faiako } \\
\text { N.PsÉ } & \text { surveiller ART homme enseigner } \\
& \text { "L'enseignant surveille." ou "L'enseignant est surveillé". }
\end{array}
$$

En futunien, si le verbo-nominal est intransitif, son actant sera aussi toujours exprimé sous une forme possessive (49a). Par contre, l'actant d'un verbonominal transitif doit garder sa marque ergative (49b), ce qui n'est pas admissible en wallisien :

$$
\begin{aligned}
& \text { (49a) e kau 'ala i le tugolo a } \\
& \text { N.PSÉ 1SG se réveiller OBL ART ronflement Poss Muni } \\
& \text { "Je suis réveillé par le ronflement de Muni." }
\end{aligned}
$$

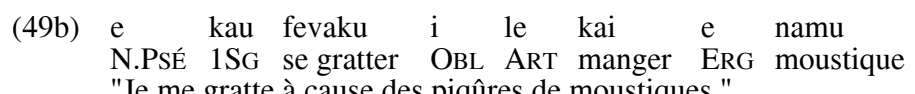

Wallisien et futunien ne rentrent pas facilement dans la typologie proposée par Koptjevskaja-Tamm (1993:61). Si l'actant S est toujours au génitif, et l'actant A toujours à l'ergatif, l'actant $\mathrm{O}$ peut soit être au génitif, soit rester à l'absolutif. Dans le premier cas, le type de nominalisation est "Ergative-Possessive" (S et O au génitif, A à l'ergatif) alors que dans le second, le type de nominalisation est étiqueté "Mixed type" (S au génitif, $\mathrm{A}$ à l'ergatif et $\mathrm{O}$ à l'absolutif).

\section{FONCTION DES FORMES NOMINALISÉES}

Clark (1981) énumère les différentes fonctions possibles pour les formes nominalisées des langues polynésiennes : prédicat dans une phrase nominale, sujet, objet, déterminant d'un nom et différents circonstants. De même en fidjien 
(Dixon 1988:134) et dans les langues kanak, il semble qu'il n'y ait aucune limite quant aux fonctions possibles. La seule exception concerne peut-être les actants à l'ergatif des langues polynésiennes (à tester)

Voici quelques exemples illustrant les possibilités d'emploi syntaxique des formes verbales nominalisées.

\subsection{Prédicat dans une phrase nominale}

Dans les récits, les phrases nominales sont très fréquentes, aussi bien pour mettre en scène les différents personnages que pour évoquer des situations ou des événements. Ces phrases nominales admettent tout à fait un prédicat verbonominal, comme dans les exemples xârâgurè suivants :
(50) kèè-mê na rè nyärä Nèpôrô mê Tuura PRÉF-venir PSÉ POSS 3PL Nèpôrô et Tuura "Les clans Nèpôrô et les Tuura sont venus."

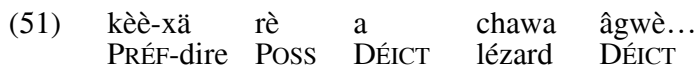
"Le lézard dit..." (comme formule d'introduction au discours direct ou indirect)

Les deux propositions d'un énoncé équatif peuvent aussi présenter des formes verbales nominalisées, comme dans l'exemple xârâcùù suivant :

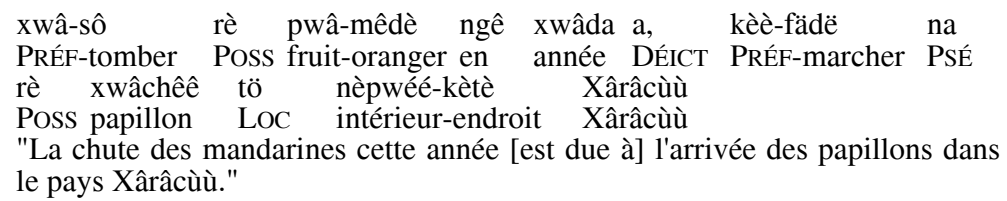

De même, en futunien, les phrases nominales, construites avec l'auxiliaire de prédication ko et ayant un centre verbo-nominal, sont extrêmement fréquentes dans les récits :
(53) ko lenā lana ifo ake ki tai PRÉD DÉICT sa descendre DIR OBL mer "Il descend vers le bord de mer." (litt. voilà sa descente vers le bord de mer)
(54) ko lenā loa le tā o le lali $\mathrm{e}$ le aloa PRÉd DÉICT SUCC ART frapper Poss ART cloche ERG ART homme "Et voilà que l'homme frappe la cloche en bois."


Des constituants à syntagme verbal nominalisé peuvent faire office de sujet ou d'objet dans les langues kanak, ou d'actant à l'absolutif dans les langues polynésiennes ergatives. Ces constructions ont le même usage que les formes infinitivales ou les complétives du français.

Futunien : actant à l'absolutif

\begin{tabular}{|c|c|c|c|c|c|}
\hline (55) & $\begin{array}{l}\text { e } \\
\text { N.PSÉ }\end{array}$ & $\begin{array}{l}\text { fakakaekae } \\
\text { fatiguer }\end{array}$ & $\begin{array}{l}\text { le } \\
\text { ART }\end{array}$ & $\begin{array}{l}\text { felōgoi } \\
\text { discuter }\end{array}$ & $\begin{array}{l}\text { mo } \\
\text { avec }\end{array}$ \\
\hline
\end{tabular}

"Discuter avec les enfants est fatigant."

Xârâcùù : sujet

(56) xêê-da rè nâ xwi, pââ-nâ wâ siè paii PrÉF-manger Poss 1SG exister dent-1SG ACCOM NÉG malade "Je peux manger, je n'ai plus mal aux dents" (litt. ma possibilité de manger existe...)

Xârâcùù : objet

(57) ngêê sati kèè-tanyûû rè chaa döu è ngû 1PLEXCL apprendre PRÉF-percer Poss un chose 3SG bouger "Nous apprenons à percer un objet qui bouge."

En wallisien, les compléments des verbes de déclaration ou de volonté, ainsi que l'interrogation indirecte introduite par la marque interrogative $p e$, se présentent préférentiellement sous forme de phrase nominale à centre verbonominal.

Les phrases nominales sont introduites par le présentatif/auxiliaire de prédication $k o$. En (58b), il s'agit d'une phrase nominale équative dont chaque proposition est introduite par ko. Comparons l'énoncé à interrogation indirecte à centre verbal ('alu "aller" instancié par la marque temporelle du passé $n e ' e$ ) en (58a) avec l'énoncé à centre verbo-nominalisé par la présence de l'article (te 'alu) en (58b), les deux énoncés étant sémantiquement équivalents :

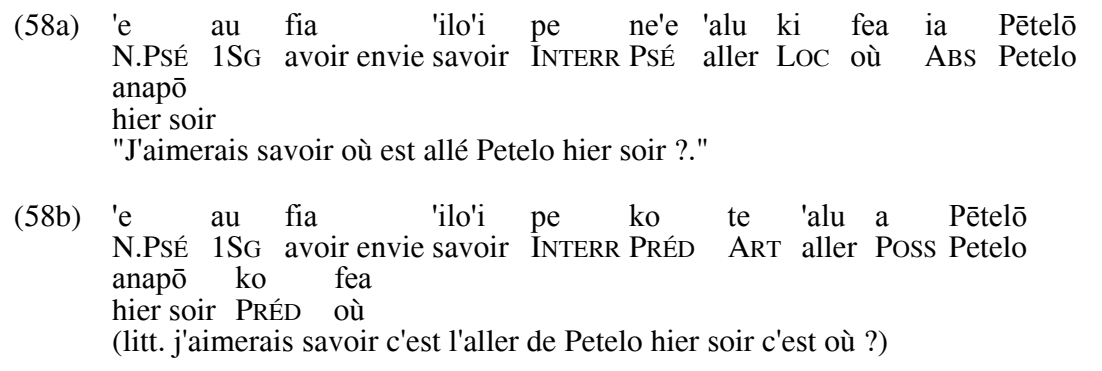




\subsection{Circonstants}

Tous les types de circonstant peuvent avoir un centre verbo-nominal. Il en va ainsi, en xârâcùù du circonstant temporel, introduit par la préposition mââitaa "avant" en (59) et par la préposition nêdee "après" en (60) :

(59) è xö mè xânî coa rè ku mââîa kèè-xûtââ 3SG bien que toujours éplucher Poss igname avant PRÉF-cuire rè ri

Poss 3PL

"Il faut toujours éplucher les ignames avant de les faire cuire."

(60) mè rè nä mûgé mètùsè rè nêdee kèè-xù

FUT 3SG INACC de nouveau dormir INACC après PRÉF-donner röö kwésùrè xù è

Poss+2SG médicament à $3 \mathrm{SG}$

"Il dormira encore après que tu lui auras donné des médicaments."

Toujours en xârâcùù, le préfixe $\grave{u}$ - nominalise des verbes ou des syntagmes verbaux qui peuvent avoir un statut de circonstant autonome, à valeur consécutive en (61) :

(61) déé cikapwèi na nôôniira, ù-châba rè dööbwi kè inondation recouvrir PSÉ plaine PRÉF-épais Poss boue venant de kètè nä

endroit DÉICT

"L'inondation a recouvert la plaine, [c'est la] raison de l'épaisseur de la boue à cet endroit."

Enfin, dans l'exemple futunien suivant, le circonstant de cause ou de raison introduit par la préposition $i$ a comme centre un lexème verbal, nominalisé par la présence de l'article spécifique le, mais qui a conservé son accord verbal en nombre marqué par la réduplication de sa première syllabe (Cf. aussi l'exemple (27):

(62)

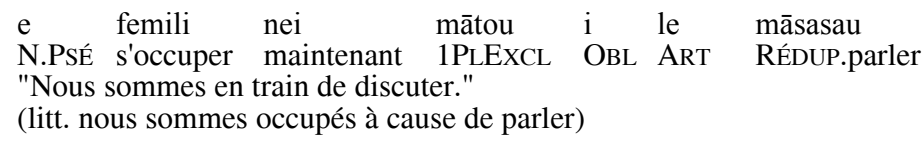

\section{CONCLUSION}

Dans les langues océaniennes mentionnées dans cet article, les formes nominalisées du verbe sont, comme dans beaucoup d'autres langues, de nature hybride, car elles présentent des caractéristiques structurelles et sémantiques typiques à la fois du verbe et du nom. Selon les langues, les constructions à verbe nominalisé présentent différents types, et ces différences sont en partie liées à la structure accusative ou ergative des énoncés. Ainsi, en xâracùù, les 
formes nominalisées sont de structure "Possessive-Accusative" si l'énoncé verbal suit l'ordre SVO, mais elles sont de type "Ergative-Possessive" lorsque l'énoncé verbal suit l'ordre VOA. En wallisien, les constructions présentent aussi différents types ("Ergative-Possessive" ou "Mixte"), selon l'ordre des constituants nominaux.

Les procédés de nominalisation diffèrent selon que la seule présence d'un article suffise, ou qu'une dérivation soit nécessaire. Dans les langues kanak, le même procédé formel de dérivation permet de créer de nouveaux lexèmes et de nominaliser des phrases verbales. Enfin, la nominalisation du verbe est compatible avec le maintien des marques aspectuelles voire parfois des marques temporelles.

\section{RÉFÉRENCES}

Bril I., 2002, Le nêlêmwa (Nouvelle-Calédonie). Analyse syntaxique et sémantique, Paris, Peeters, Langues et cultures du Pacifique 16.

Chung S., 1973, The syntax of nominalizations in Polynesian, Oceanic Linguistics 12 p. 641-86.

-, 1978, Case Marking and Grammatical Relations in Polynesian, Austin, University of Texas Press.

Clark R., 1981, Inside \& outside Polynesian nominalizations, in Hollyman J. and Andrew Pawley (eds), Studies in Pacific Languages and Cultures in Honour of Bruce Biggs, Auckland, Linguistic Society of New Zealand, p. 65-81.

Dixon R.M.W., 1988, A Grammar of Boumaa Fijian, Chicago-London, The University of Chicago Press.

Hooper R., 1996, Type and instance nominalisations in Tokelauan, in Lynch, J. \& Fa'afo Pat (eds), Oceanic studies: Proceedings of the First International Conference on Oceanic Linguistics, Canberra, The Australian National University, Pacific Linguistics C-133, p. 223-241.

Koptjevskaja-Tamm M., 1993, Nominalizations, London and New York, Routledge, Theoretical Linguistics.

Moyse-Faurie C, 1995, Le xârâcù̀̀, langue de Thio-Canala (NouvelleCalédonie). Eléments de syntaxe, Peeters-Selaf, Langues et Cultures du Pacifique 10.

- 1997, Grammaire du futunien, Nouméa, Centre de Documentation Pédagogique, coll. Université, 240p.

- 2000, Possessive markers in East Uvean (Faka'uvea), Sprachtypologie und Universalienforschung. Language Typology and Universals, Berlin 53:3/4, p. 319-332.

- 2005, Problèmes de catégorisation syntaxique dans les langues polynésiennes, in Lazard G. et Moyse-Faurie C., Linguistique typologique, Lille, Presses du Septentrion, p. 161-192.

Rivierre J.-C., 1980, La langue de Touho. Phonologie et grammaire du cèmuhî (Nouvelle-Calédonie), Paris, Selaf, Langues et civilisations à tradition orale $\mathrm{n}^{\circ} 38$.

Vernaudon J. et B. Rigo, 2004, De la translation substantivante à la quantification : vers une caractérisation sémantique de l'article te en tahitien, Bulletin de la Société de Linguistique de Paris 99, p. 457-480. 